\title{
Espace biofonctionnel et mise en condition tissulaire : quelles perspectives en prothèse amovible complète ?
}

\section{RÉSUMÉ}

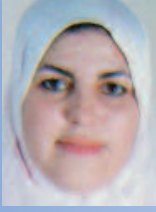

Anissa REGRAGUI

Résidente en prothèse amovible, Faculté de médecine dentaire de Rabat, Université Mohamed V,

BP 6212,

Rabat - Instituts, Maroc.

\section{Ahmed ABDEDINE}

Professeur d'enseignement supérieur, Chef de service de prothèse amovible au centre de consultation

et des traitements dentaires

de Rabat-CCTD,

Faculté de médecine dentaire de Rabat, Université Mohamed V,

BP 6212,

Rabat-Instituts, Maroc.

\section{Nadia MERZOUK}

Professeur d'enseignement supérieur en prothèse amovible

Faculté de médecine dentaire de Rabat, Université Mohamed V,

BP 6212,

Rabat-Instituts, Maroc.

Face à une fibromuqueuse lésée ne pouvant plus assurer son rôle visà-vis du tissu osseux et à des organes périprothétiques ayant diminué l'espace potentiel réservé à la prothèse, une mise en condition tissulaire, plus ou moins longue, s'impose dans la majorité des cas. Cette remise en condition tissulaire devra répondre à certains critères pour être efficace : rétablissement d'une dimension verticale correcte, réalisation d'une occlusion balancée et bases prothétiques suffisamment étendues pour soutenir le matériau de mise en condition tissulaire.

Les résines plastiques à prise retardée utilisées dans ce type de traitement ont pour but d'assurer une répartition harmonieuse des charges occlusales afin de permettre la guérison des tissus lésés et I'obtention d'une extension des surfaces d'appui secondaires [1, 2].

L'objectif de cet article est de mettre l'accent, à travers un cas clinique, sur l'aspect thérapeutique des arcades complètement édentées et fortement résorbées présentant une altération de l'espace biofonctionnel, ce qui met en jeu la rétention et la stabilité des prothèses complètes, surtout mandibulaires.

espace biofonctionnel

- mise en condition tissulaire

- résines plastiques à prise retardée

- résorption osseuse

stomatite prothétique
AOS 2011;255:197-206

DOI: $10.1051 /$ aos/2011303

(C) AEOS / EDP Sciences 


\section{Introduction}

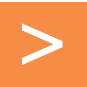

Les qualités fondamentales des tissus de revêtement des surfaces d'appui maxillaire et mandibulaire et des organes paraprothétiques jouent un rôle primordial dans la sustentation, la stabilisation et la rétention des prothèses amovibles.

Elles jouent, en outre, un rôle secondaire non négligeable dans l'établissement d'une dimension verticale correcte et dans l'enregistrement d'une relation centrée stable et satisfaisante.

Elles participent enfin à la préservation de l'intégrité des structures osseuses et musculaires de l'appareil stomatognathique d'un édenté complet.

Chez l'édenté complet, la sphère orale présente de profondes modifications, directement liées à l'édentation elle-même, à l'âge de l'édentation ainsi qu'à l'âge de l'individu [3].

L'édentation totale s'inscrit en effet dans le processus du vieillissement et se manifeste par des modifications anatomiques et physiologiques locorégionales, liées à la perte des dents et de l'os alvéolaire. L'incidence esthétique, psychologique et fonctionnelle est constante.

La sénescence altère toutes les structures anatomiques et physiologiques et, en particulier, les surfaces d'appui. La résorption de l'os crestal et la migration relative des insertions des muscles mylohyoïdiens et buccinateurs vers le sommet de la crête résiduelle sont sources de difficultés dans la réalisation de prothèse adjointe, surtout à la mandibule [4, 5] (fig. 1).

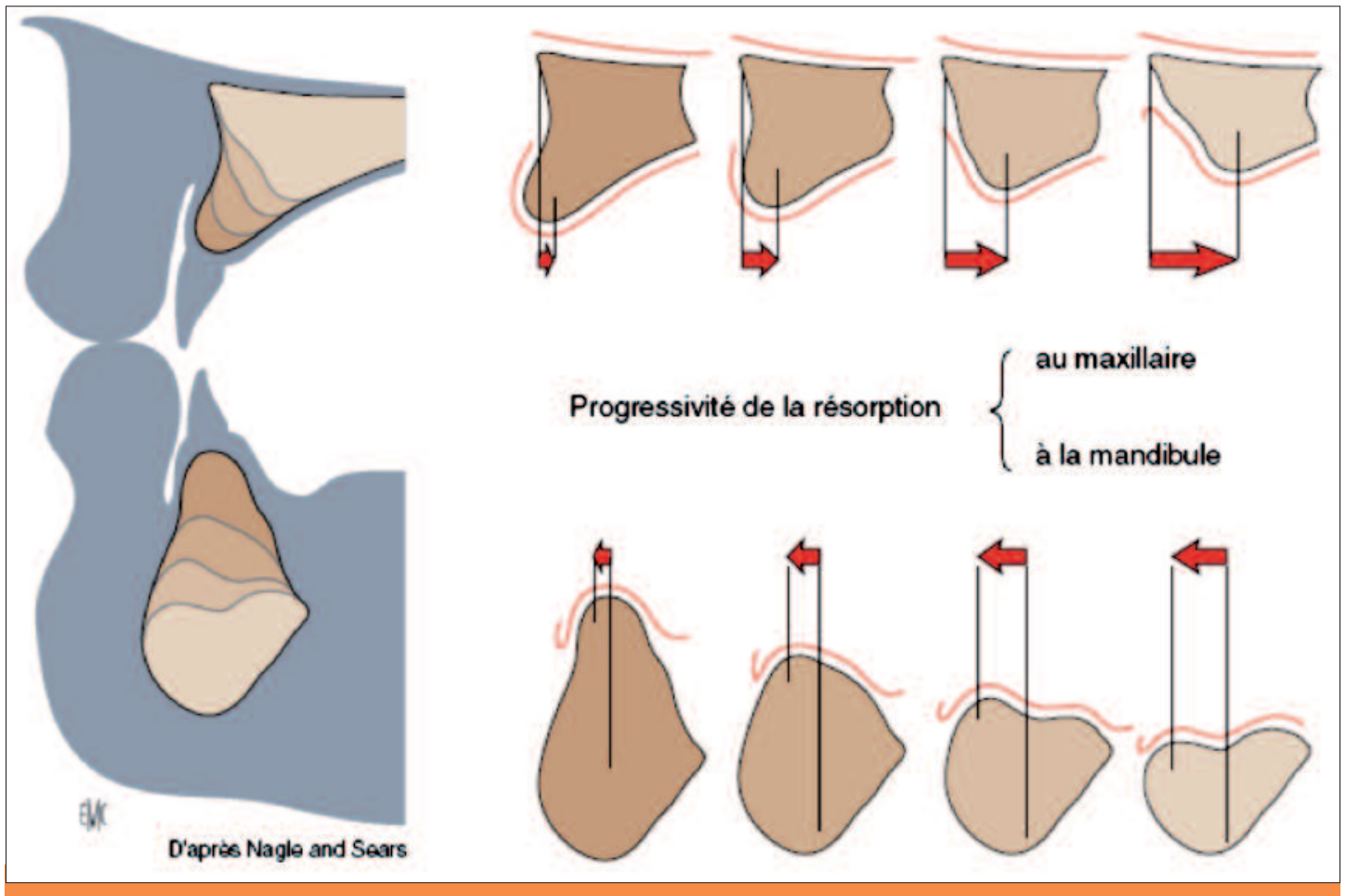

Fig. 1 Description selon Nagle and Sears de la résorption des maxillaires après extractions dentaires [5]. 
La muqueuse s'amincit et les muscles perdent leur tonicité. De plus, en vieillissant, l'ensemble du système neurovégétatif s'altère.

D'autre part, le port d'une prothèse amovible complète de conception erronée, instable ou mal équilibrée, mal adaptée, entraîne des altérations tissulaires supplémentaires : comprimées ou lésées, la fibromuqueuse et la muqueuse perdent leur épaisseur physiologique et leur viscoélasticité [6].

Elles n'adhèrent plus aux plans profonds, et elles ne peuvent plus jouer leur double rôle bénéfique de stimulation des tissus osseux sousjacents et d'amortisseur des pressions exercées au cours des principales fonctions de mastication et de déglutition.

Devant ces conditions, il apparaît nécessaire de tenter une réhabilitation de l'ensemble des structures de la cavité orale et des articulations temporo-mandibulaires ainsi que de préparer psychologiquement les patients à recevoir leurs prothèses. C'est dans ce cadre que s'inscrit le rôle de la mise en condition à l'aide des résines acryliques à prise retardée.

\section{Présentation d'un cas}

\section{État initial}

Mme H.Z., âgée de 68 ans, consulte pour une restauration prothétique amovible pouvant remplacer sa prothèse ancienne défectueuse et délaissée par la patiente il y a trois ans.

\section{> Examen exobuccal}

III révèle un affaissement de l'étage inférieur de la face, ce qui a pour conséquences l'accentua- tion des plis nasogéniens, une perte de projection labiale et une proéminence du menton (fig. 2 a et b).

\section{$>$ Examen endobuccal}

L'examen endobuccal révèle des surfaces d'appui très résorbées avec dédoublement du fond du vestibule bien marqué au niveau du maxillaire supérieur ; le palais présente l'empreinte irritative du système ventouse de l'ancienne

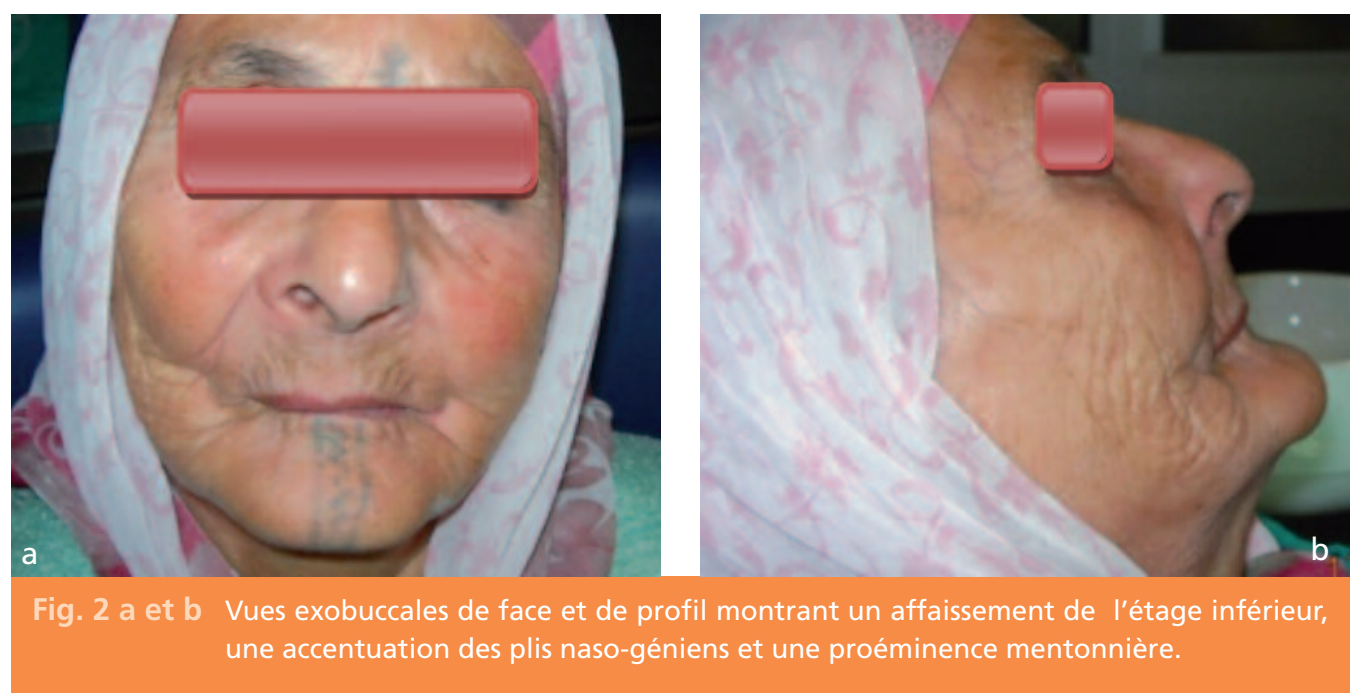


prothèse amovible. Une ulcération est par ailleurs localisée à la jonction palais dur-palais mou (fig. 3 a à c).

À la mandibule, la résorption importante met à nu les deux apophyses Géni, les surfaces d'appui étant très résorbées (fig. 4).

\section{> Examen des prothèses}

Les prothèses sont sous-dimensionnées, tous les bords prothétiques sont sous-étendus :
- la prothèse maxillaire présente au niveau de I'intrados un système ventouse métallique pour améliorer la rétention ;

- la prothèse mandibulaire quant à elle, ne recouvre pas les apophyses Géni.

Placées en bouche, les deux prothèses ne montrent aucun respect des règles de réalisation rigoureuse de prothèse complète : dimension verticale d'occlusion, relation centrée, plan d'occlusion et bords prothétiques erronés.
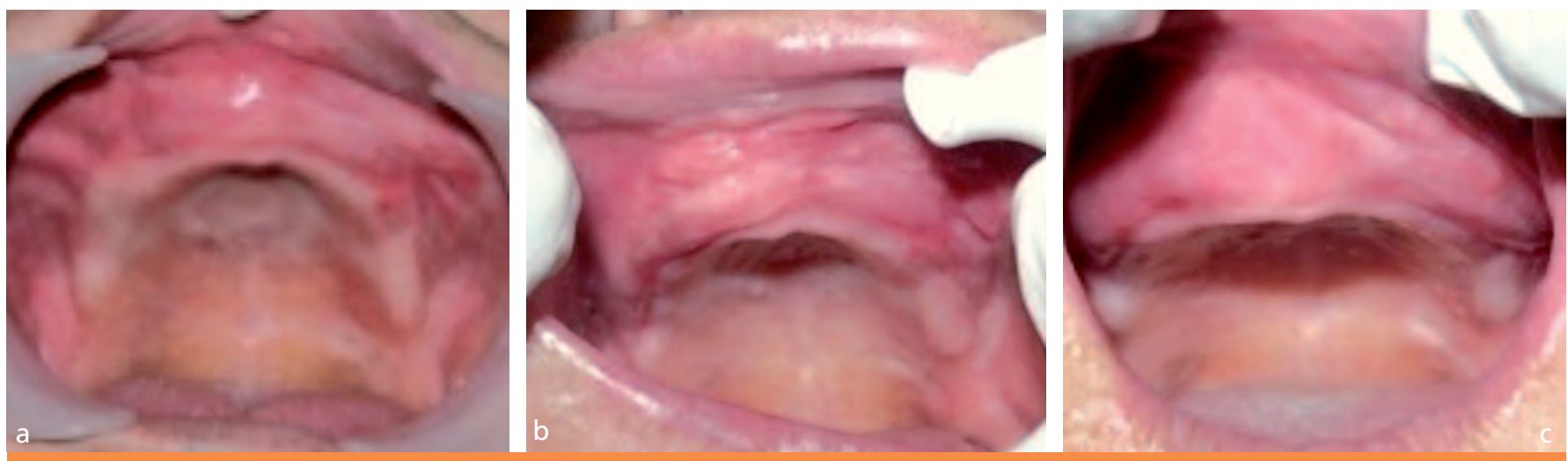

Fig. 3 a à c Vues endobuccales du maxillaire supérieur montrant une crête très résorbée avec dédoublement du fond du vestibule, l'empreinte du système ventouse de l'ancienne prothèse amovible, ainsi qu'une ulcération à la jonction palais dur-palais mou.

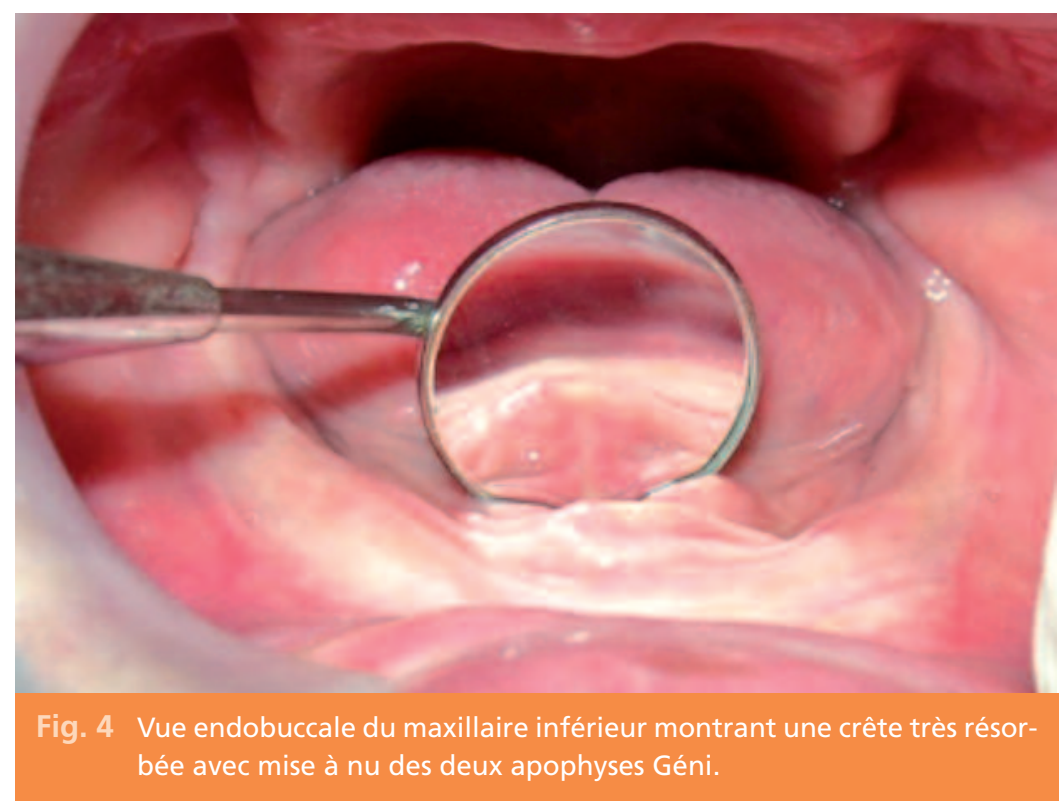




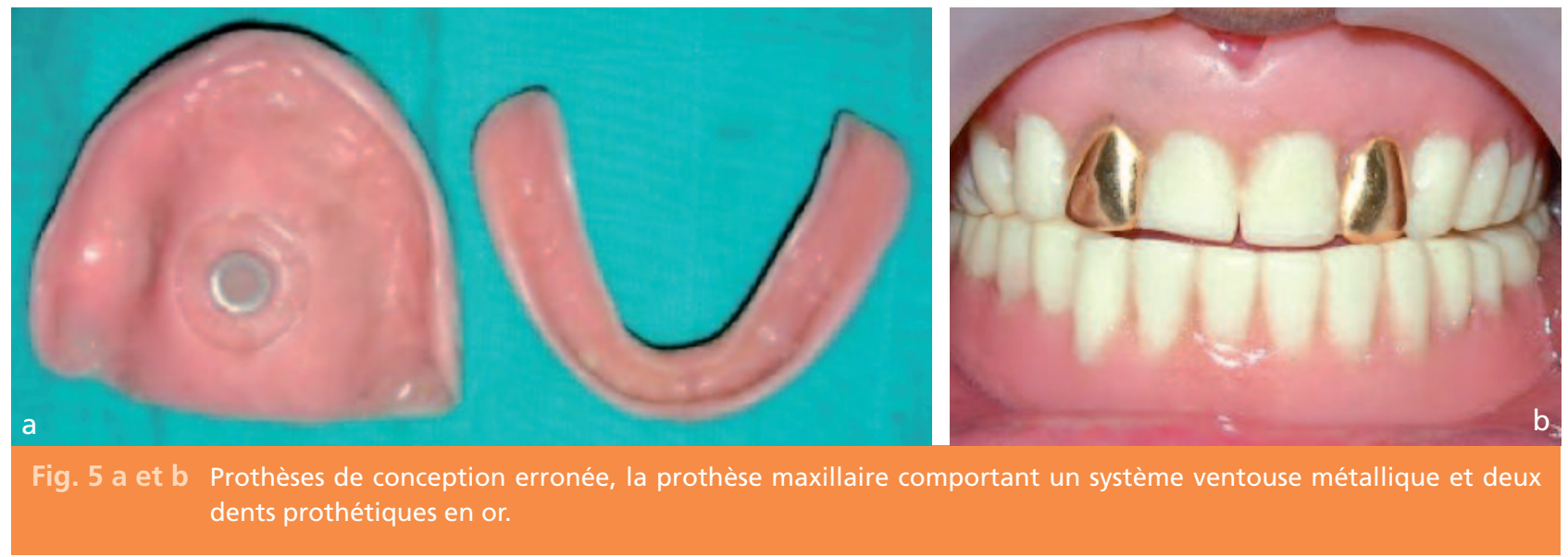

La prothèse supérieure comporte deux dents prothétiques en or (fig. 5 a et b).

\section{Objectifs du traitement}

Vu la conception erronée des prothèses existantes, les surfaces d'appui s'en trouvent altérées, rendant indispensable la réalisation de nouvelles prothèses amovibles en passant par des séances de mise en condition tissulaire.

\section{$>$ Intérêts de mise en condition tissulaire $[7,8,9]$}

La mise en condition tissulaire permet de :

- favoriser le comportement histologique, morphologique et physiologique des surfaces d'appui en contact avec l'intrados prothétique ;

- améliorer l'intégration psychique et organique de la nouvelle prothèse ;

- augmenter l'espace biofonctionnel et, par conséquent, réaliser une empreinte correcte exploitant toutes les zones anatomiques favorables à l'équilibre prothétique ; I'espace biofonctionnel étant la zone dans laquelle la prothèse doit s'inclure harmonieusement sans interférer avec les muscles environnants.

\section{> Application}

La méthode fait appel à des produits spécifiques, résines à prise retardée et prothèses dont l'intrados est adapté à ce type de traitement (anciennes prothèses ou nouveaux appareils).

L'étendue des surfaces d'appui primaire et secondaire doit être correcte, les bords (joints périphériques) sont étendus et soutenus par modelage avec une résine autopolymérisante dans les cas d'insuffisance, ou replacés par soustraction dans les cas d'hyperextension.

L'action se situe à deux niveaux distincts [10, 11] :

- directement sur la muqueuse : I'élimination de la stomatite prothétique ou d'autres lésions permet au corps de régénérer ces tissus. Le résultat est une muqueuse saine cliniquement ;

- indirectement sur l'os, les forces s'exerçant sur ce tissu dur sont tempérées par les produits résineux de traitement, et permettent une organisation osseuse capable structurellement de répondre efficacement à des actions principalement masticatoires. Ce fait est particulièrement évident en prothèse transitoire post-extractionnelle où ce type de 
traitement conserve un volume donc une valeur positive.

\section{Étapes de réalisation}

\section{> Réalisation d'une prothèse transitoire maxillaire}

\section{- Empreintes}

Dans un premier temps, une empreinte primaire de l'arcade maxillaire est prise avec de I'alginate pour réaliser un porte-empreinte individuel; puis l'empreinte maxillaire secondaire est réalisée avec un polysulfure de basse viscosité de type Permlastic Light ${ }^{\circledR}$.

\section{- Enregistrement du rapport maxillo-mandibulaire}

Une maquette d'occlusion maxillaire en résine munie de bourrelets en stents est préparée puis stabilisée sur son modèle avec de l'oxyde de zinc-eugénol.

La prothèse du bas a été rectifiée par des ajouts de résine au niveau des zones présentant des manques.

La maquette d'occlusion est ensuite réglée en situation clinique : le plan d'occlusion de la maquette supérieure, parallèle au plan de Camper, est contrôlé à l'aide d'une règle de Fox, puis le transfert du moulage maxillaire est effectué sur l'articulateur. La dimension verticale d'occlusion (DVO) est évaluée et le moulage inférieur muni de la prothèse amovible corrigée est monté sur articulateur après enregistrement de la relation centrée $(\mathrm{RC})$ à la bonne DVO (fig. 6 b).

La prothèse transitoire réalisée présente des plans de morsure au niveau du secteur postérieur du fait de la difficulté du guidage vers la relation centrée (fig. 6 a).

\section{> Mise en condition neuromusculaire et neuro-articulaire (fig. 6 c)}

Le traitement de mise en condition neuromusculo-articulaire préalable vise, en effet, à [10, 12] :

- rétablir une dimension verticale correcte (sa diminution provoque un proglissement mandibulaire et son augmentation entraîne une surcharge articulaire) ;

- guider la mandibule en relation centrée ;

- rétablir une occlusion correcte par l'intermédiaire de plans de morsure qui vont permettre de déconnecter tous les réflexes acquis d'occlusion erronée en rapport avec le port d'anciennes prothèses ;
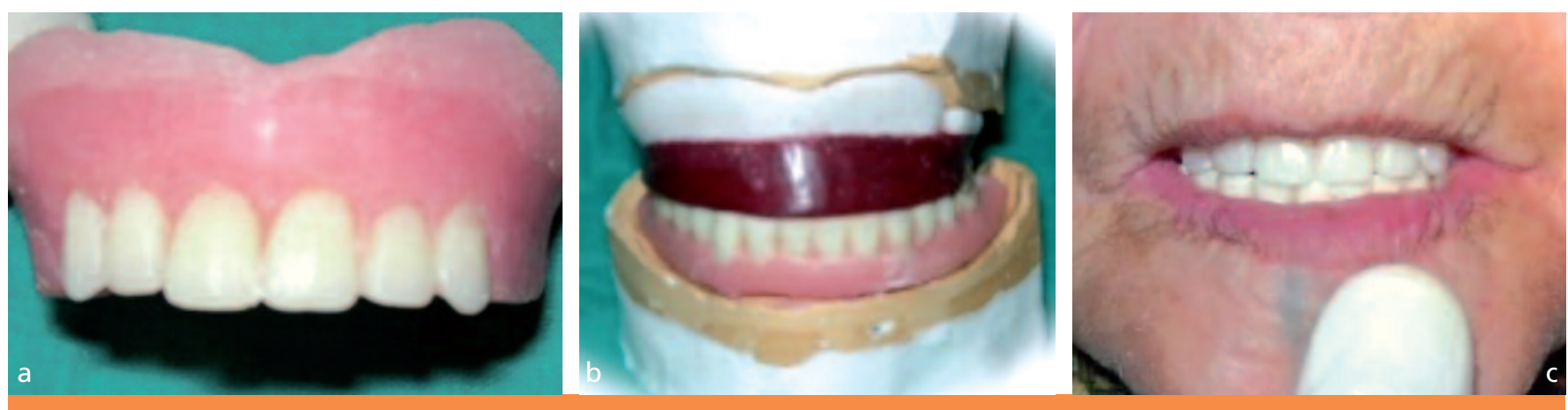

Fig. 6 a à c Réalisation d'une prothèse transitoire maxillaire présentant des plans de morsure au niveau du secteur postérieur du fait de la difficulté du guidage vers la relation centrée. 
- relâcher les muscles et corriger les trajectoires condyliennes asymétriques.

\section{> Mise en condition tissulaire}

Un certain nombre de produits sont sur le marché, parmi lesquels : Coe Comfort de Coe, Fitt de Kerr, Hydrocast de Kay See Dental MFG, Viscogel de De Trey. Ils se présentent sous la forme poudre et liquide. La poudre est un polyméthylméthacrylate ou un copolymère d'éthylméthacrylate ou d'isobutylméthacrylate $[8,13]$.

Le liquide est composé d'éthanol et d'esters aromatiques. Ces esters agissent comme des plastifiants et sont le plus souvent du dibutylphtalate. Le mélange liquide-poudre donne une solution fluide dont la viscosité augmente au fur et à mesure que se produit la pénétration éthanol-plastifiant. Le matériau devient alors suffisamment visqueux pour être inséré en bouche ( 2 à 3 minutes après le début du mélange). Il atteint l'état de gel en 15 à 20 minutes.

À ce stade, la plasticité apparaît et elle disparaîtra progressivement dans le milieu buccal pour faire place à des caractéristiques d'élasticité. Ce processus est lié à la perte d'éthanol et à l'adsorption d'eau.

Cinq phases se succèdent $[8,14]$ :

- phase physique : mélange poudre-liquide ;

- phase chimique : début de la polymérisation ;

- phase plastique active : insertion de la prothèse et enregistrement d'une empreinte (cette dernière phase dure environ 10 minutes) ;

- phase élastique : amortissement des pressions au niveau des tissus de soutien (phase pouvant durer de 15 jours à 3 semaines) ;

- phase de dessiccation granuleuse : apparaît simultanément à la perte d'élasticité et conduit à la destruction de l'état de surface du produit.

Dans notre cas, le matériau utilisé est le Fitt de Kerr qui présente une fluidité initiale, une élasticité instantanée faible et une déformation retardée tout en absorbant les contraintes occlusales $[14,15]$.

L'évaporation de l'alcool contenu dans le matériau et l'éclatement de bulles d'air emprisonnées rendent le Fitt de Kerr poreux, dur et irritant ; en plus il favorise la prolifération microbienne d'où l'intérêt de changer le matériau tous les 4 à 8 jours régulièrement (fig. 7 a).

Après trois mois de mise en condition tissulaire, nous avons remarqué une nette amélioration des surfaces d'appui par la disparition de tout signe d'inflammation ou d'ulcération.

En plus, l'espace biofonctionnel devient plus important surtout au niveau maxillaire où un bon approfondissement vestibulaire est remarqué (fig. 7 b et c).

\section{> Empreintes différées}

Une empreinte différée est réalisée sur les prothèses existantes, rebasées périodiquement par des produits souples dont le principe est d'exercer, pendant un certain temps, une action modelante par la modification progressive de surface [5].

L'état de surface de l'intrados est amélioré grâce à un lavis de silicone fluide (Permlastic Light ${ }^{\circledR}$ ) (fig. 8).

\section{$>$ Transfert sur articulateur}

Une fois que les empreintes sont coulées, nous procédons au transfert précis des moulages de travail sur articulateur par engrènement des deux prothèses mémorisant la DVO et le RMM préalablement validés (fig. 9). 

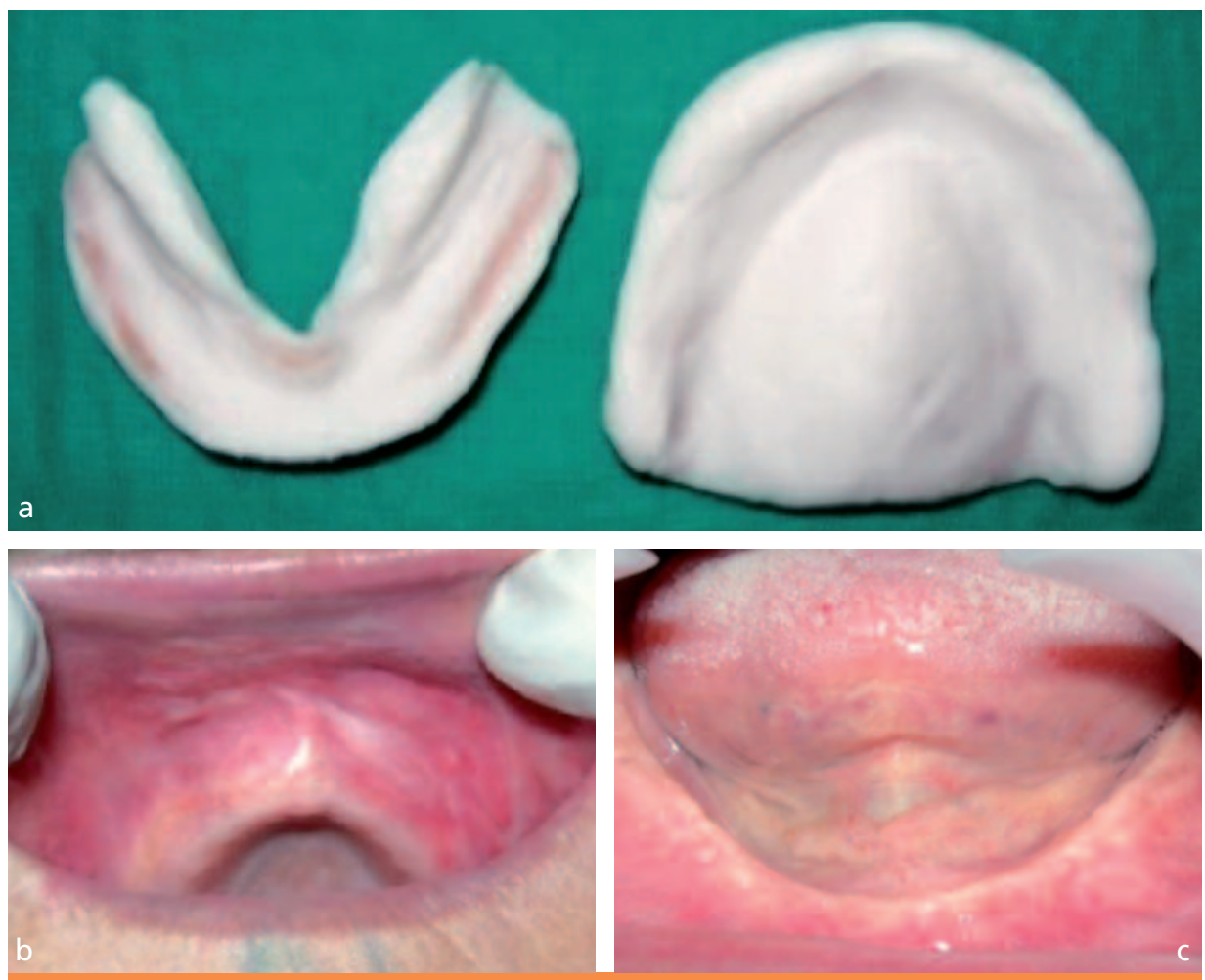

Fig. 7 a à c Après 3 mois de mise en condition tissulaire, l'espace biofonctionnel est nettement augmenté avec disparition de tout signe d'inflammation ou d'ulcération.
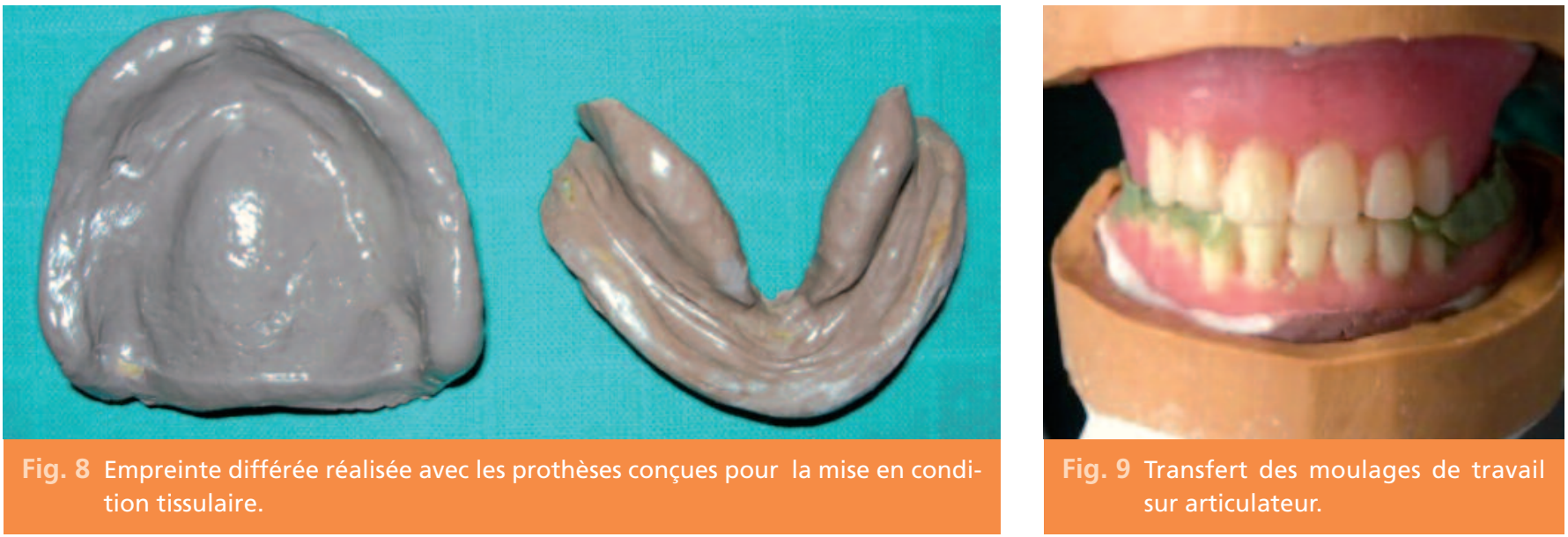

Fig. 9 Transfert des moulages de travail sur articulateur.

\section{$>$ Montage des dents et finition des cires}

La mise en place des dents prothétiques doit respecter le concept de l'occlusion intégralement équilibrée (fig. 10).

\section{$>$ Pose de la prothèse en bouche}

Après polymérisation, les prothèses sont essayées en bouche, révélant une nette amélioration de la rétention et de la stabilité prothé- 
tiques grâce à l'augmentation remarquable de l'espace biofonctionnel.

La patiente, une fois les nouvelles prothèses en place, témoigne sa satisfaction du résul- tat obtenu tant sur les plans esthétique et fonctionnel que sur les plans stabilité-rétention prothétiques, par son large sourire (fig. 11).

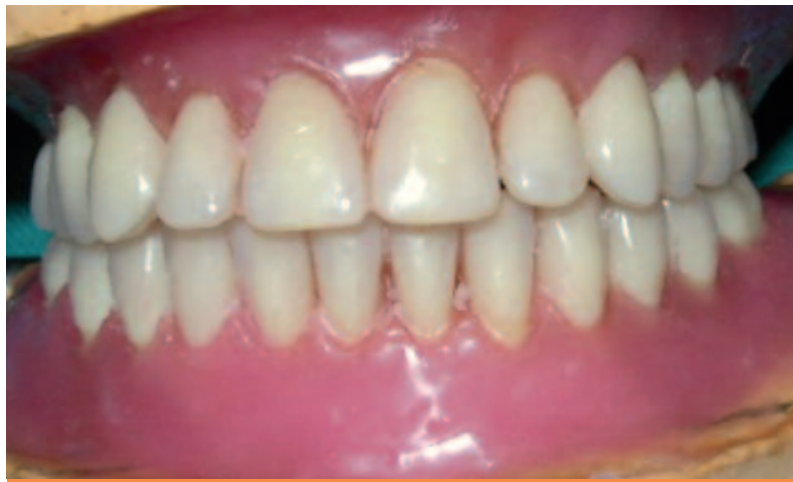

Fig. 10 Montage des dents prothétiques.

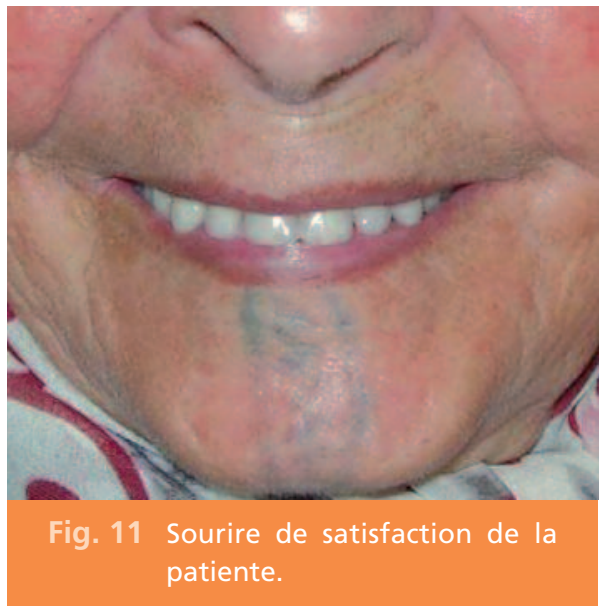

\section{Conclusion}

La mise en condition tissulaire semble un chemin obligé sur la voie du succès en prothèse amovible complète ; elle permet d'élaborer harmonieusement un lit prothétique adéquat en améliorant la qualité des surfaces d'appui en rapport avec la restauration prothétique et en augmentant l'espace réservé à la prothèse par un bon approfondissement vestibulaire, ce qui aboutit à un état cliniquement satisfaisant, dans les meilleures conditions de temps et d'efficacité remplaçant ainsi les gestes chirurgicaux soustractifs.

\section{Bibliographie}

1. Benfdil F, Merzouk N, Rahmani EM, Benamar A, Abdedine A. Intérêt de la mise en condition tissulaire dans la remise en état des muqueuses buccales. Act Odonto Stomatol 2003;222:139-50.
2. Guimelli B, Hunger $F$. Le problème de l'état des muqueuses maxillaires et mandibulaires avant, pendant et après l'insertion des prothèses totales. Chir Dent Fr 1989; 471/472:61-71.

3. Le Bars P, Giumelli B, Daniel A.
Vieillissement et stomatite prothétique (analyse stéréologique). Clinic 1995;16(1):51-2.

4. Taddei C, Wolfram-Gabel R, Waltmann E. Prothèse amovible : anatomie de l'édenté total. Encycl Med Chir, Stomat Odont II, 1995; 


\section{3-325-A-10:10p.}

5. Jaudoin $\mathrm{P}$, Millet $\mathrm{C}$, Mifsud S.

Empreintes en prothèse complète. Encycl Med Chir, Odontologie, 2006; 23-325-C-10:19p.

6. Le Bars $P$, Amouriq $Y$, Bodic F, Giumelli B. Réactions tissulaires au port de prothèse dentaire amovible partielle ou totale.

Encycl Med Chir, Odontologie, 2002; 23-325-P-10:10p.

7. Chevaux JM, Nanfi C, Tosello A, Preckel E, Pesci-Bardon C. Les résines à prise retardée : utilisation rationnelle en fonction de leurs propriétés physico-chimiques. Cah Prothèse 2000;111:43-53.

8. Grimonster J.
Les conditionneurs

tissulaires et leurs emplois

en prothèse adjointe :

applications cliniques.

Act Odonto Stomatol

1997; 200:655-69.

9. Lejoyeux J, Lejoyeux R. Mise en condition en prothèse amovible. Paris: Masson, 1993:23-73.

10. Jaudoin $P$, Millet $C$, Jaudoin $\mathrm{E}$.

Traitements préprothétiques chez l'édenté total. Encycl Med Chir, Odontologie, 2007, 23-325-C-05: $5 \mathrm{p}$.

11. Besse F, Dupuis V. Mise en condition tissulaire non chirurgicale en prothèse adjointe complète.

Clinic 2002;23:319-24.

12. Lassauzay $C$, Veyrune $\mathrm{JL}$, Lesher J.

La préparation des surfaces d'appui : rôles de la prothèse complète amovible transitoire.

Cah Prothèse 1998;104:79-87.

13. Lejoyeux J, Lejoyeux R. Mise en condition tissulaire dans le traitement d'une édentation totale mandibulaire.

Act Odonto Stomatol 1992;177:69-81.

14. Grimonster J.

Les conditionneurs tissulaires et leurs emplois en prothèse adjointe : composition et propriétés physico-chimiques. Actual Odonto Stomatol 1996;196:581-92.

15. Grimonster J, Brogniez V. Entretien des prothèses amovibles : revue non exhaustive des méthodes et produits proposés. Act Odonto Stomatol 1989;167:619-32.

\section{SUMMARY}

\section{Biofunctional space and tissular preparation:} what perspectives in complete removable prosthesis?

Anissa REGRAGUI, Ahmed ABDEDINE, Nadia MERZOUK

\section{Keywords \\ - space out biofonctionnel - prepared tissular - plastic resins with delayed grip - osseous reduction - prosthetic stomatitis}

In front of a hurt masticatory, not being able to any more assure its role face to face some bone tissue ; in front of organs periprosthetic having decreased the potential space reserved for the prosthesis, a tissular, more or less long preparation, is imperative in the majority of the cases. This discount in tissular condition will have to answer certain criteria to be effective: restoring of a correct vertical dimension, a realization of a rocked occlusion and base prothétiques enough spread to support the material of tissular preparation.

Plastic resins with delayed grip used in this type of treatment aim at assuring a harmonious distribution of occlusales loads to allow the cure of hurt fabrics and obtaining of an extension of the secondary surfaces of support. The objective of this article, is to emphasize, through a clinical case on the therapeutic aspect of completely broken the teeth and strongly reduced arches presenting a change of the biofoctionnel space what involves the keeping back and the stability of the complete prostheses, especially mandibular. 